\title{
Libro Healthy Towns - assessing availability of food information for a pilot public health initiative
}

\section{Abstract}

In Ireland SI 489/2014 mandates food businesses (FB) to present written allergen information on food and drink at the point of presentation or sale. Despite this requirement being in place since 2014, compliance is low. A 2017 audit published by the Food Safety Authority Ireland reported that corrective action was required by $88 \%$ of FB assessed.

Calorie labeling, although not legally required, has strong consumer demand. Furthermore when FB implement calorie labeling, improvements to stock management resulted in reduced costs.

This study aims to establish a baseline of available information to evaluate the progress of the public health initiative "Libro Healthy Towns": a pilot project aimed at supporting FB to provide food information to consumers.

A catchment area was established to identify FB for inclusion. FB were categorised by business type: Multi-site (MB) or Independent site (IB), and service type: Restaurant/Café (RC), Restaurant/Takeaway (RT), Hotel, Pub/Restaurant and Takeaway. Availability of allergen and calorie information were collected by observing and photographing food information on display. Where information was not observable, it was requested. Employees were asked if consumers requested calorie information. Responses were recorded for input into a spreadsheet. Statistical analysis was conducted using SPSS (ver. 24). Results were assessed using Chi-Square and Likelihood Ratio.

In total $54 \mathrm{FB}$ were assessed $(31=\mathrm{MB}, 23=\mathrm{IB}), 63 \%$ had allergen information displayed and $31 \%$ had it available on request. There was no statistically significant difference between business type and allergen information being available on display or by request. Three businesses $(6 \%)$ had no allergen information available; $100 \%$ of these were IB. MB were significantly more likely to have allergen information available $(100 \%)$ compared with IB $(87 \%)$ (p-value $=.021)$. Calorie information was available for $24 \%$ $(\mathrm{n}=13)$ of $\mathrm{FB}$, of which significantly more $(92 \%)$ were $\mathrm{MB}(\mathrm{p}$-value $=.003)$. Calorie information was requested in $56 \%$ of $\mathrm{FB}$, most frequently requested in RC and RT, 62\% and 55\% respectively. Significantly more MB reported that consumers requested calorie information compared with IB, $77 \%$ and $26 \%$ respectively ( $\mathrm{p}$-value $=<.001$ ).

Only half of businesses displayed allergen information in writing at the point of presentation or sale. Consumer interest in calorie information was strong, supporting previous research showing consumers want to make informed food choices. Access to food information was easier in MB however this study shows that measures to improve the availability of food information are necessary across all business types.

\section{Conflict of Interest}

The Authors F. Douglas and D.O'Kelly provided supervision from Nutritics, a private commercial company. 